\title{
Synthesizing radar maps of polar regions with a Doppler-only method
}

\author{
Mark S. Roulston and Duane O. Muhleman
}

\begin{abstract}
A method for producing a radar-reflectivity map of the polar regions of the Moon or a planet from polar orbit with only the frequency shift of the reflected signals is described and simulated. A Radon transform of the reflectivity is obtained during multiple passes over the pole. Inversion of this Radon transform enables a map of radar reflectivity to be synthesized. (C) 1997 Optical Society of America

Key words: Radon transform, Doppler radar, polar radar map.
\end{abstract}

\section{Introduction}

The most common technique used for imaging in planetary radar astronomy is delay-Doppler imaging, which makes use of both the time delay and the frequency shift of the reflected signal to reconstruct the spatial variation in reflectivity of the target surface. This technique has been used to produce radar images from Earth of the Moon, ${ }^{1}$ Mercury, ${ }^{2}$ Venus, ${ }^{3}$ and Mars. ${ }^{4}$ Delay-Doppler imaging can also be performed by orbiting spacecraft. The most sophisticated method for delay-Doppler imaging is that of synthetic aperture radar, which has been used to image the Earth ${ }^{5}$ as well as Venus during the Magellan mission. ${ }^{6}$

An alternative to delay-Doppler imaging is the use of only the Doppler shifts of the reflected signals when cw spectra of the target are obtained for transverse velocities over a range of angles, as described by Thomson and Ponsonby. ${ }^{7}$ When the contours of constant Doppler shifts can be approximated as straight lines, the measured data and the reflectivity distribution are related by a two-dimensional Fourier transform. ${ }^{8}$

This paper presents a method for reconstructing radar-reflectivity maps of polar regions from low polar orbit with Doppler shifts only. In the low-orbit case the contours of constant Doppler shift are hyperbolas. Such maps can be synthesized by use of

\footnotetext{
The authors are with the division of Geological and Planetary Sciences, California Institute of Technology 150-21, 1201 E. California Boulevard, Pasadena, California 91125.

Received 15 July 1996; revised manuscript received 21 January 1997.

0003-6935/97/173912-08\$10.00/0

(C) 1997 Optical Society of America
}

repeated passes over the pole as the planet rotates and then the application of a modified Radon transform inversion operator to recover the reflectivity distribution. It should be stressed that, although this method for imaging poles is simpler than the methods described above, it will yield inferior results when compared with delay-Doppler imaging or synthetic aperture radar.

Although the original motivation for this work was to devise a low-cost system to obtain radar maps of the Lunar poles in an effort to locate ice, the method could be applied to the polar regions of any of the terrestrial planets, particularly Mercury.

First we describe the principle of the method and show that the method produces a Radon transform of the spatial variation of reflectivity around the pole. We then present an algorithm for inverting this transform, allowing the reflectivity distribution to be recovered. We then present the results, which demonstrate the method's feasibility even when realistic levels of noise are added to the data, of numerical simulations of the method.

\section{Description of the Method}

The geometry of the method is shown in Fig. 1. Consider a spacecraft at $O$ flying at an altitude $H=S O$ above a flat surface. Let the direction of motion be the $x$ axis and let $\mathbf{v}$ be the velocity vector of the spacecraft. Let the origin of the coordinate system be centered on the spacecraft. The Doppler shift of a signal reflected from a point on the surface at position $\mathbf{r}=\mathbf{O A}$ depends on the point's velocity relative to the spacecraft projected onto the line joining the point to the spacecraft. If we call this velocity the Doppler 


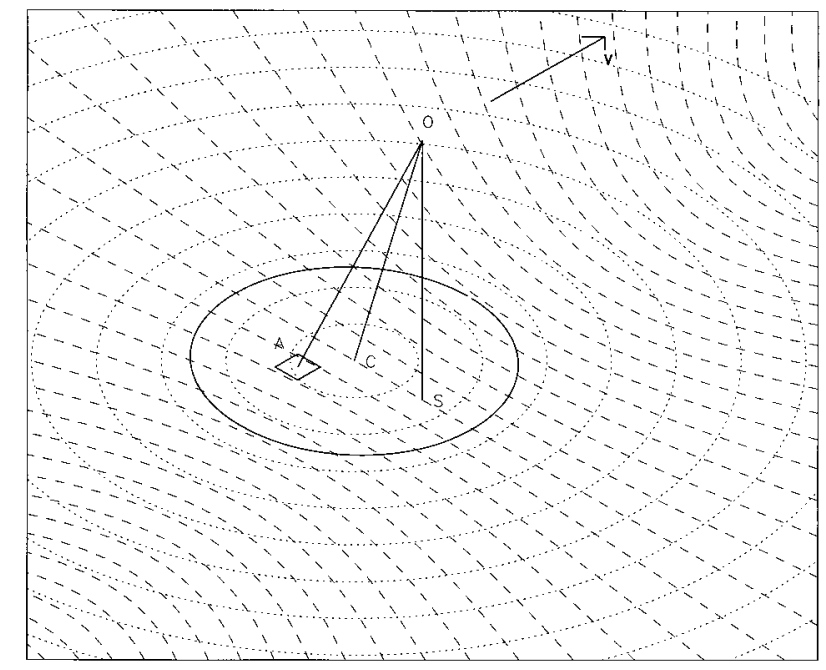

Fig. 1. Geometry of the method. The spacecraft is located at $O$, directly above the subspacecraft point $S$. The point $C$ is the pole of the planet, and the radar beam axis is along $O C$. The solid ellipse is the beam footprint. The dotted curves are lines of latitude (although the polar region is approximated here as a plane), and the dashed curves are the contours of constant Doppler shift. The spacecraft velocity is given by the vector $\mathbf{v}$.

velocity, $v_{d}$, then

$$
v_{d}=-\mathbf{v} \cdot \frac{\mathbf{r}}{|\mathbf{r}|} .
$$

The vectors $\mathbf{v}$ and $\mathbf{r}$ are given by $\mathbf{v}=[v, 0,0]$ and $\mathbf{r}=$ $[x, y,-H]$, respectively, where $x$ and $y$ are the coordinates of the reflecting point on the ground. Equation (1) can therefore be written as

$$
v_{d}=-\frac{x v}{\left(x^{2}+y^{2}+H^{2}\right)^{1 / 2}} .
$$

The resulting Doppler shift is given by

$$
\delta v=-\frac{2 v_{d} v_{0}}{c}
$$

where $v_{0}$ is the frequency of the transmitted signal, $c$ is the speed of light, and the factor 2 arises because it is a reflection with both the outbound and the inbound propagation contributing to the Doppler shift. Rearranging Eq. (2) gives

$$
y^{2}=x^{2}\left(v^{2} / v_{d}^{2}-1\right)-H^{2},
$$

which says that the contours of constant Doppler shift on the surface are hyperbolas. form

Equation (4) can be written in the dimensionless

$$
Y^{2}=X^{2} / V^{2}-1,
$$

where $X$ and $Y$ are in units of the orbital altitude $H$ and $V$ is given by

$$
V=\left(v^{2} / v_{d}^{2}-1\right)^{-1 / 2}
$$

In this form $V$ is the value of the $X$ intercept of the hyperbola. It is useful to make a change of coordinates from $(X, Y)$ to $(V, \gamma)$, where

$$
\begin{aligned}
& X=V \cosh \gamma, \\
& Y=\sinh \gamma .
\end{aligned}
$$

In this new system the dimensionless area element is given by

$$
\mathrm{d} X \mathrm{~d} Y=\cosh ^{2} \gamma \mathrm{d} V \mathrm{~d} \gamma .
$$

The total power reflected with frequencies inside the frequency interval that corresponds to $V \rightarrow V+\Delta V$ is therefore

$$
\Delta P(V)=\int_{V}^{V+\Delta V} \mathrm{~d} V^{\prime} \int_{-\infty}^{+\infty} \mathrm{d} \gamma \mu(X, Y) W(X, Y) H^{2} \cosh ^{2} \gamma
$$

where $X$ and $Y$ are functions of $V^{\prime}$ and $\gamma$ given by Eqs. (7) and (8), $\mu(X, Y)$ is the reflectivity of the surface, and $W(X, Y)$ is a weighting function that incorporates beam effects, the phase function, and geometrical factors. The $H^{2}$ factor is to give a dimensioned area. Differentiation of Eq. (6) relates $\mathrm{d} V$ to $\mathrm{d} v_{d}$ :

$$
\mathrm{d} V=\left(\frac{v^{2}}{v_{d}^{3}}-1\right)^{-3 / 2} \frac{v^{2}}{v_{d}^{3}} \mathrm{~d} v_{d}=V^{3} \frac{v^{2}}{v_{d}^{3}} \mathrm{~d} v_{d} .
$$

Next, $W$ is expressed in terms of physical quantities. Let $\theta$ be the angle between $\mathbf{r}$ and the vertical, that is, $\theta=A \hat{O} S$, and let $\phi$ be the angle between $\mathbf{r}$ and the axis of the beam, that is, $\phi=A \hat{O} C$. The monostatic radar equation for the power received from an element of area $\mathrm{d} A$ is

$$
\mathrm{d} P_{r}=\frac{P_{t}(\phi) A_{e}^{2} \mu \cos (\theta) F(\theta)}{\lambda^{2} R^{4}} \mathrm{~d} A,
$$

where $P_{t}(\phi)$ is the transmitted power per unit solid angle, $\lambda$ is the wavelength, $R$ is the distance between the spacecraft and the area element, that is, $R=|\mathbf{r}|=$ $O A, A_{e}$ is the antenna area, and $F$ is the scattering function. The value of $R$ is given by

$$
R^{2}=x^{2}+y^{2}+H^{2}
$$

and $\theta$ and $\phi$ can be obtained by use of

$$
\begin{aligned}
\cos \theta & =H / R, \\
\cos \phi & =\frac{x x_{0}+y y_{0}+H^{2}}{R\left(x_{0}{ }^{2}+y_{0}{ }^{2}+H^{2}\right)^{1 / 2}},
\end{aligned}
$$

where $\left(x_{0}, y_{0}\right)$ are the coordinates of $A$, the point on the surface at which the beam is aimed. The scattering function $F$ is related to the surface roughness at the wavelength in question. For a circularly polarized signal the scattering function used for reflection with the opposite-sense circular polarization 
(OC) was the one given by Muhleman ${ }^{9}$ :

$$
F(\theta)=K_{1} \frac{\alpha \cos \theta}{(\sin \theta+\alpha \cos \theta)^{3}} .
$$

The parameter $\alpha$ is related to surface roughness, while $K_{1}$ is a normalization factor. Appropriate values at a wavelength of $3.5 \mathrm{~cm}$ for the Moon are $\alpha=0.4$ and $K_{1}=2.4821$.

The scattering function used for the same-sense (SC) circular polarization was

$$
F(\theta)=\frac{3}{2 \pi} \cos ^{2} \theta
$$

Comparison of Eq. (10) with Eq. (12) indicates that

$$
W(X, Y)=\frac{P_{t}(\phi) A_{e}^{2} F(\theta) \cos (\theta)}{R^{4} \lambda^{2}},
$$

with $R, \theta$, and $\phi$ given by Eqs. (13)-(15), respectively, and $H^{2}\left(Y^{2}+1\right)$ as the $H^{2} \cosh ^{2} \gamma$ area element. Thus the measured quantity will be $\Delta P\left(v_{d}\right)$, given by Eq. (10), with the appropriate substitutions from Eqs. (7), (8), (11), (13)-(15), and (18). If the spacecraft beam is pointing at the pole of the Moon and the spacecraft is in a low Lunar orbit, then on the next orbital pass the Moon will have rotated through approximately $1^{\circ}$ under the spacecraft. If we take the coordinate system to be fixed in the frame of the spacecraft, then the function $\mu(X, Y)$ must be replaced by its rotation through the azimuthal angle $\alpha$ in Eq. (10):

$$
\Delta P\left(v_{d}, \alpha\right)=\int_{V}^{V+\Delta V} \mathrm{~d} U \int_{-\infty}^{+\infty} \mathrm{d} \gamma W(X, Y) \mathscr{T} \alpha[\mu(X, Y)]
$$

where $\mathscr{T}_{\alpha}$ is a rotation through $\alpha$. Equation (19) actually represents a two-dimensional Radon transform. ${ }^{10}$ This transform takes a function $g(x, y)$ and converts it to a function $\mathscr{R} g(p, \alpha)$, which is the line integral of $g$ along a line parameterized by $p$ and $\alpha$. These line integrals are usually along straight lines; $\alpha$ is the angle between a reference axis and the normal to the line, and $p$ is the perpendicular distance between the origin and the line. Equation (19) is different in that the line integrals are performed along hyperbolas where $V$ is the $x$ intercept of a hyperbola and $\alpha$ is the angle between the $x$ axis and some reference line. During a period of 14 days the Moon will complete a half-rotation and $\alpha$ will range from 0 to $\pi$. Any additional orbits will not increase the amount of information because $\Delta P\left(v_{d}, \alpha\right)$, for values of $\alpha$ between $\pi$ and $2 \pi$, is equal to $\Delta P\left(-v_{d}, \alpha-\pi\right)$.

The ground resolution of the technique is determined by the distance between contours of constant Doppler shift that correspond to $v$ and $v+\Delta v$, where $\Delta \nu$ is the resolvable frequency interval. The approx- imate distance between these contours $\Delta x$ is given by

$$
\Delta x \approx \frac{H}{2} \frac{\Delta v}{v_{0}} \frac{c}{v} .
$$

The resolvable frequency $\Delta v$ is approximately the reciprocal of the duration of the signal obtained while the spacecraft is positioned above the point $S$. A signal of $\sim 1$-ms duration is required to achieve a frequency resolution of $1 \mathrm{kHz}$. During this time the spacecraft will move less than $2 \mathrm{~m}$ with respect to the ground.

\section{Inversion Problem}

The observation that Eq. (19) represents a form of Radon transform is very useful when one inverts the data, represented by $\Delta P\left(v_{d}, \alpha\right)$, to recover the reflectivity map of the surface represented by $\mu(X, Y)$. The inversion of the Radon transform has been much studied in the context of medical tomography. ${ }^{11}$ The inversion method used was that of convolution. The convolution kernel used is provided by Nievergelt. ${ }^{12}$ The corresponding inversion formula is

$$
\begin{aligned}
g(x, y)= & \lim _{q \rightarrow 0} \frac{1}{\pi} \int_{0}^{\pi} \mathrm{d} \alpha \int_{-\infty}^{+\infty} \mathrm{d} p \\
& \times \mathscr{R} g(p+x \cos \alpha+y \sin \alpha, \alpha) G_{q}(p),
\end{aligned}
$$

where $G_{q}$ is a convolution kernel given by

$$
G_{q}(p)= \begin{cases}\frac{1}{\pi q^{2}} & |p| \leq q \\ \frac{1}{\pi q^{2}}\left[1-\left(1-q^{2} / p^{2}\right)^{-1 / 2}\right] & |p|>q\end{cases}
$$

It can be shown that the integral of $G_{q}$ is a regularizing family $\rho_{A}$, where $A=1 / q$. This is another way of saying that, as $A$ tends to infinity (or $q$ tends to zero), the convolution of a function with $\rho_{A}$ tends to the Hilbert transform of that function; consequently, in the limit $q \rightarrow 0$, Eq. (21) is equivalent to

$$
g(x, y)=-\frac{1}{2 \pi} \mathscr{R} \cdot \mathscr{H} \cdot \mathscr{D} \cdot \mathscr{R} g
$$

where $\mathscr{D}$ represents partial differentiation with respect to $p$ and $\mathscr{H}$ represents the Hilbert transform. Equation (23) is a form of the inversion formula given by Radon. ${ }^{13}$

When we evaluate Eq. (21) numerically, $q$ must have some finite value. $G_{q}$ has its most weight around $p=0$. This indicates simply that, when we evaluate $g(x, y)$, most weight is given to values of $\mathscr{R} g(p, \alpha)$ when the line represented by $(p, \alpha)$ passes close to the point $(x, y)$. Therefore Eq. (21) can be modified for the case when $(p, \alpha)$ represents hyper- 
bolas:

$$
\begin{aligned}
g(x, y)= & \lim _{q \rightarrow 0} \frac{1}{\pi} \int_{0}^{\pi} \mathrm{d} \alpha \int_{-\infty}^{+\infty} \mathrm{d} p \\
& \times \operatorname{Rg}\left\{p \frac{x \cos \alpha+y \sin \alpha}{\left[(y \cos \alpha-x \sin \alpha)^{2}+1\right]^{1 / 2}}, \alpha\right\} G_{q}(p) .
\end{aligned}
$$

If we assume that the weighting function $W(X, Y)$ is circularly symmetric (which would correspond to a vertically incident beam), then it can be folded with $\mu(X, Y)$ in Eq. (19), and the inversion formula is given by

$$
\begin{aligned}
L(X, Y)= & \lim _{q \rightarrow 0} \frac{1}{\pi} \int_{0}^{\pi} \mathrm{d} \alpha \int_{-\infty}^{+\infty} \mathrm{d} v \\
& \times \Delta P\left\{V+\frac{X \cos \alpha+Y \sin \alpha}{\left[(Y \cos \alpha-X \sin \alpha)^{2}+1\right]^{1 / 2}}, \alpha\right\} \\
& \times G_{q}(V),
\end{aligned}
$$

where

$$
L(X, Y)=\mu(X, Y) W(X, Y) .
$$

Of course, in practice the integration over $V$ cannot be infinite and will be limited by the total bandwidth of the receiver; also the data will be discrete rather than continuous. A discrete form of Eq. (25) is

$$
\begin{aligned}
L(X, Y)= & \frac{1}{M} \sum_{i=1}^{M} \sum_{j=-N}^{j=+N} \Delta V_{j} \Delta P_{i j} G_{q} \\
& \times\left(V_{j}+\frac{X \cos (i \Delta \alpha)+Y \sin (i \Delta \alpha)}{\left\{[Y \cos (i \Delta \alpha)-X \sin (i \Delta \alpha)]^{2}+1\right\}^{1 / 2}}\right),
\end{aligned}
$$

where $\Delta P_{i j}$ is a matrix element containing the value in frequency bin $j$ received during orbital pass $i$ and $\Delta \alpha$ is the change in the azimuthal angle between successive orbits. $V_{j}$ and $\Delta V_{j}$ are vectors that contain the values and increments of the parameter $V$ that corresponds to the $j$ th frequency interval. $M$ is the number of orbits, and $N$ is related to the total bandwidth of the receiver. The required bandwidth can be derived from Eqs. (1) and (2) and is given by

$$
\text { bandwidth }=\frac{4 v_{0} v \theta_{\max }}{c\left(1+\theta_{\max }^{2}\right)^{1 / 2}},
$$

where $\theta_{\max }$ is the half-width of the beam in which there is appreciable power. The number of frequency bins is then given by the bandwidth divided by the frequency resolution $\Delta \nu$. Equation (27) assumes that the data were taken at equal angular intervals from 0 to $\pi$. After $L(X, Y)$ has been obtained, the known weighting function $W(X, Y)$ can be divided out to obtain $\mu(X, Y)$.

If $W(X, Y)$ is not circularly symmetric, as will be the case if the beam is pointed obliquely at the surface, $\mu$ and $W$ can no longer be folded together because in Eq. (19) $\mu(X, Y)$ is rotated while $W(X, Y)$ is not. The problem of inverting a Radon transform with a generalized weighting function $W(X, Y, \alpha)$ has been addressed by Kunyansky ${ }^{14}$ in the context of emission tomography. Kunyansky derived the following iterative formula to reconstruct the original function:

$$
\epsilon^{(k+1)}(X, Y)=\epsilon_{D}(X, Y)+\mathscr{R}^{-1}\left(\mathscr{R}-\mathscr{R}_{W}\right) \epsilon^{k}(X, Y),
$$

where $\epsilon_{D}(X, Y)$ represents the distorted image obtained by application of the regular Radon inversion $\mathscr{R}^{-1}$ to the weighted Radon transform represented by $\mathscr{R}_{W}$. The first term in the series, $\epsilon^{0}$, is an arbitrary function. Equation (29) is then applied iteratively to remove the distortion in $\epsilon_{D}$ caused by the asymmetry of the weighting function. Before applying the $\mathrm{Ku}$ nyansky algorithm we should note that a beam aimed at several tens of degrees off the vertical still has a large amount of circular symmetry about the beam axis. The weighting function $W$ can be written as a product of a circularly symmetric part and a noncircularly symmetric part. That is, in polar coordinates,

$$
W(r, \theta)=S(r) Q(r, \theta) .
$$

There are of course an infinite number of functions that satisfy Eq. (30), but $S$ should be chosen such that $Q$ is as close as possible to unity. The function $S$ that does this is given by

$$
S(r)=\frac{\left\langle W^{2}\right\rangle}{\langle W\rangle},
$$

where $\langle\ldots\rangle$ represents averaging over $\theta$. The function $S$ can be folded with $\mu$ and divided out after the inversion as before, and the Kunyansky algorithm can be used to remove the residual distortion that is due to $Q$. Another way to use the substantial circularly symmetric component in the weighting function is to expand the weighting function as a Fourier series in which only low-order terms will be significant. This approach was followed by Kunyansky.

\section{Simulation and Inversion}

We simulated data sets by numerically evaluating Eq. (19) and then inverting the simulated data, using the discrete version of the Nievergelt inversion formula [Eq. (27)]. The function $(\sin 8 \phi / 8 \phi)^{2}$ was used for the beam function; this was merely for computational convenience and a Bessel function or an arbitrary nonsymmetric function could be used.

In the real world, noise will be present in the data. Four sources of noise were included in the simulations:

(1) Spacecraft orientation drift: On each orbital pass over the pole it would be ideal if the beam axis were directed exactly at the pole. In reality, on each pass there is a small deviation of the beam axis, typically of the order of a few degrees. This devia- 
(a)

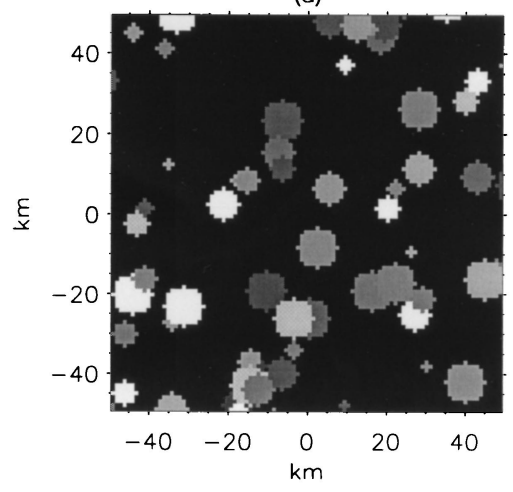

(c)

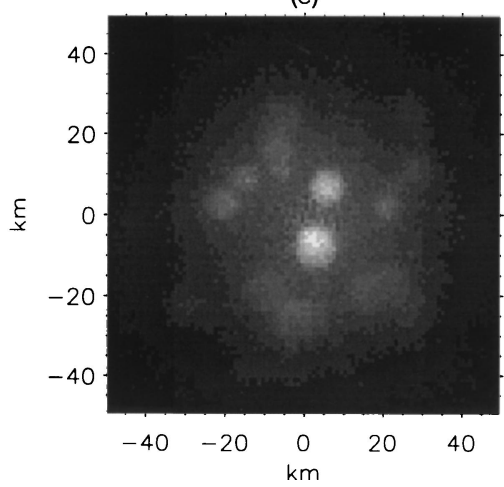

(b)

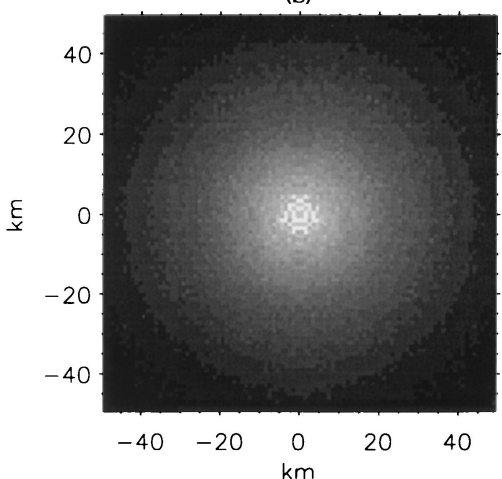

(d)

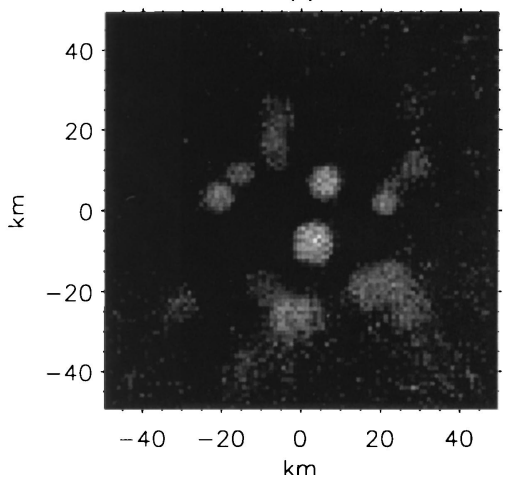

Fig. 2. Results of inverting the simulated data for OC: (a) the target surface, (b) the OC weighting function, (c) the result of inversion before correcting for the weighting function, and (d) the result of inversion after dividing out the weighting function.

tion has the effect of shifting the weighting function with respect to the pole. The contours of constant Doppler shift depend only on the spacecraft's position and velocity and are not affected by changes of orientation.

(2) Orbital altitude drift: Owing to nonzero multipole terms in the gravitational potential of the target body (for example, those caused by lunar mascons) the orbital altitude drifts. This leads to variations in the weighting function and also has the more serious effect of shifting the width and the positions of the Doppler strips on the ground.

(3) Thermal noise: There is system noise arising from the finite temperature of the receiver $T_{R}$. The rms value of this noise is given by

$$
P_{N}=k_{B} T_{R} \Delta \nu,
$$

where $k_{B}$ is Boltzmann's constant and $\Delta v$ is the resolvable frequency interval.

(4) Quantization noise: As well as being sampled discretely in time and space, the power is also discretely sampled in magnitude. If 8-bit digitization is assumed, measurements of power are quantized into integer values between 0 and 255. The difference between the true, continuous value and the digitized, rounded value is a form of noise.

We simulated the orientation drift and the altitude drift by introducing random perturbations to the beam axis direction and orbital height. The pertur- bations were changed for each orbital pass and had a Gaussian distribution. After a data set had been simulated, Gaussian thermal noise with a rms value given by Eq. (32) was added to it. The data were then quantized, the values of power being binned in 256 bins.

Data sets were simulated with two phantom surfaces that represented $\mu_{\mathrm{OC}}$ and $\mu_{\mathrm{SC}}$. These surfaces are shown in Figs. 2(a) and 3(a), respectively. The mean orbital altitude was $150 \mathrm{~km}$ with a standard deviation of $5 \mathrm{~km}$. The radar power was $10 \mathrm{~W}$, the center frequency was $8.6 \mathrm{GHz}(\lambda=3.5 \mathrm{~cm})$, and the frequency resolution was $1 \mathrm{kHz}$. The area of the transmitting-receiving antenna was $7.85 \times 10^{-3} \mathrm{~m}^{2}$. The spacecraft velocity was $1.6 \mathrm{~km} \mathrm{~s}^{-1}$. The halfwidth of the beam that contained appreciable power was taken to be $45^{\circ}$; thus Eq. (28) gives a required bandwidth of $114 \mathrm{kHz}$, although a bandwidth of 200 $\mathrm{kHz}$ was actually used in the simulation and inversion. The beam was targeted vertically downward, but a wobble with a standard deviation of $3.2^{\circ}$ was incorporated. The standard deviation of the added thermal noise corresponded to a receiver temperature of $1000 \mathrm{~K}$. With these mission parameters relation (20) predicts a ground resolution of $\Delta x \approx 1.6$ $\mathrm{km}$.

Figure 2(a) shows the reflectivity map for the opposite-sense polarization, that is, it is a gray-scale representation of $\mu_{\mathrm{OC}}(X, Y)$. Figure 2(b) shows the weighting function used for the OC case; it includes geometrical factors and the beam profile, but it is 
(a)

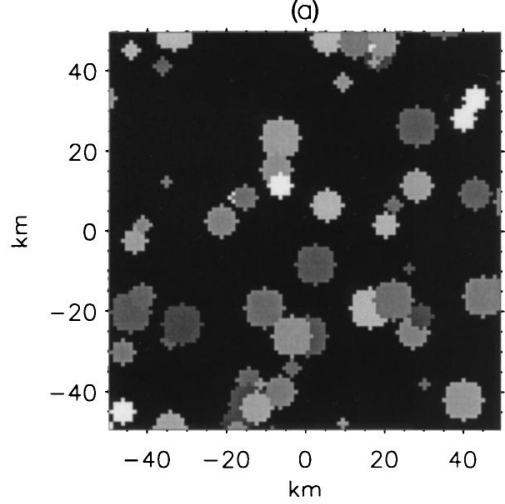

(c)

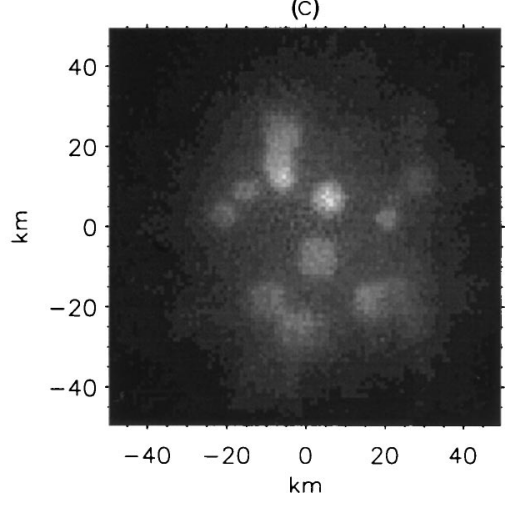

(b)

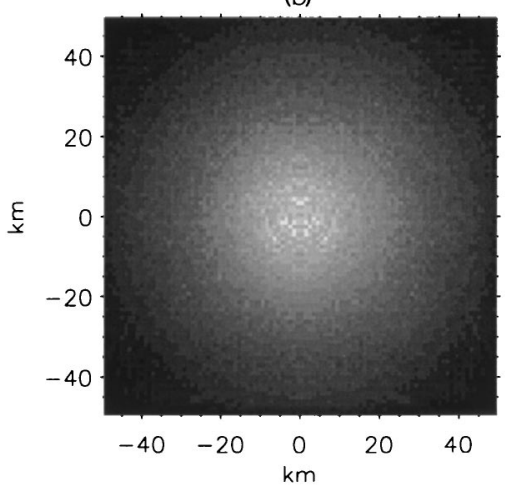

(d)

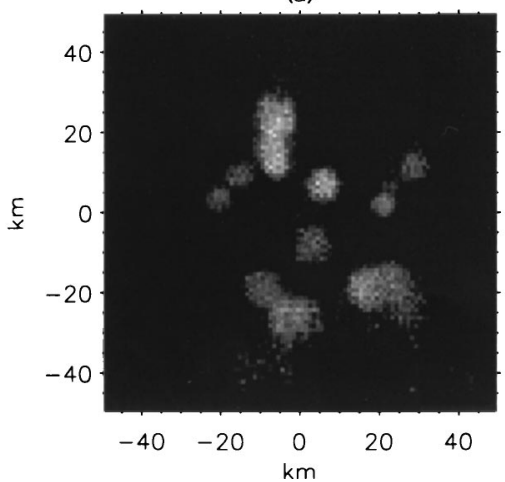

Fig. 3. Results of inverting the simulated data for SC: (a) the target surface, (b) the SC weighting function, (c) the result of inversion before correcting for the weighting function and (d) the result of inversion after dividing out the weighting function.

dominated by the sharp peak of the Muhleman scattering function. Figure 2(c) shows the image obtained by the inversion of the simulated data that had thermal noise added to it, corresponding to a receiver temperature of $1000 \mathrm{~K}$. The optimum value of the parameter $q$ in the convolution kernel was found to be 0.671 , and this value was used for all inversions. The weighting function was then divided out to obtain Fig. 2(d). Figures 3(a)-3(d) show the corresponding images for the case of SC polarization. Note that the $\mathrm{SC}$ weighting function lacks the specular peak found in the OC case.

Ice found on solar system bodies often has an anomalously high $\mu_{\mathrm{SC}} / \mu_{\mathrm{OC}}$ ratio. ${ }^{15}$ When one attempts to locate ice, it is this reflectivity ratio that must be determined. Figure 4(a) shows the ratio of Figs. 3(a) and 2(a). The scale in Fig. 4(a) has been stretched so that only regions for which $\mu_{\mathrm{SC}} / \mu_{\mathrm{OC}}>$ 0.8 are shown. The bright region slightly off center in the upper-left quadrant has a high $\mu_{\mathrm{SC}} / \mu_{\mathrm{OC}}$ value typical of cold, cracked ice. Figure 4(b) is the value of $\mu_{\mathrm{SC}} / \mu_{\mathrm{OC}}$ obtained from the inversions stretched in the same way as for Fig. 4(a). The bright region in the upper-left quadrant is clearly visible, as are the two bright regions in the lower-left and lower-right quadrants.
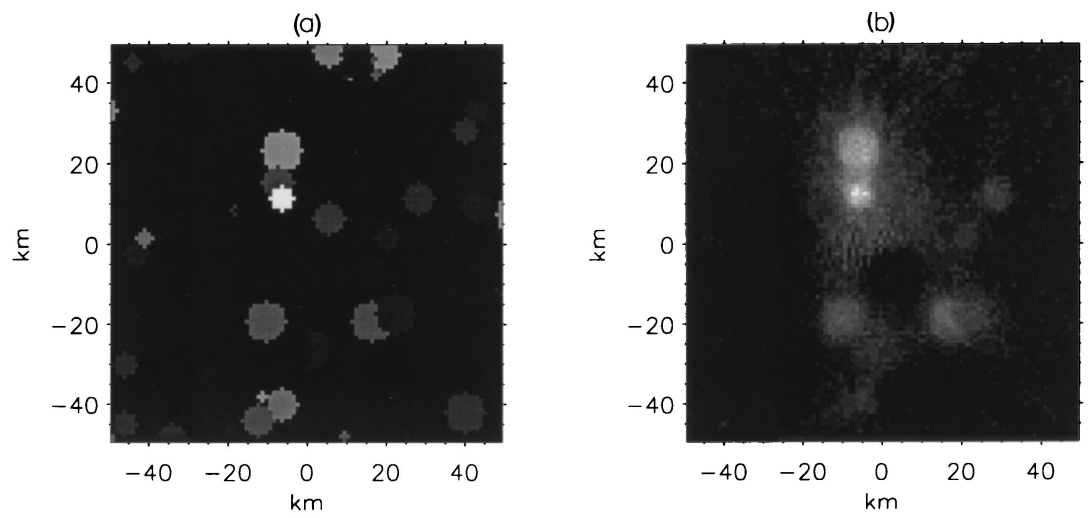

Fig. 4. Ratio of surface reflectivity in the OC and SC: (a) the ratio for the target surfaces and (b) the ratio inferred from the inversions of simulated data. In both cases the maps have been scaled so that only values of $\mu_{\mathrm{SC}} / \mu_{\mathrm{OC}}$ greater than 0.8 are shown. 


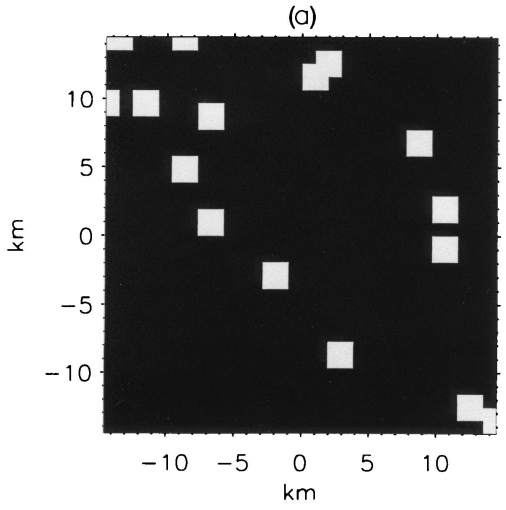

(b)

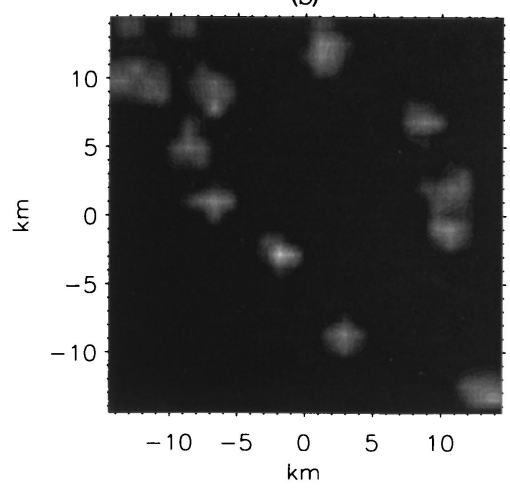

Fig. 5. Results of a resolution test: (a) The central $900 \mathrm{~km}^{2}$ of a target surface designed to test resolution and (b) the result of inversion and beam correction. This result indicates that the resolution of the method is approximately $1 \mathrm{~km}$ for the mission parameters used (orbital altitude of $150 \pm 5 \mathrm{~km}$, nadir pointing with a $3.2^{\circ}$ standard deviation in orientation, radar power of $10 \mathrm{~W}$, and a noise temperature of $1000 \mathrm{~K})$.

To obtain the results shown in Figs. 2-4 we made no attempt to correct for altitude drift and beam pointing drift. To measure the resolution of the technique we used as the target surface a surface of randomly distributed squares with a side length of 2 $\mathrm{km}$ and reflectivities of unity. The central $900 \mathrm{~km}^{2}$ of this surface is shown in Fig. 5(a). The data set that would be obtained from such a surface was then simulated with the same mission parameters as for the above figures. The result of inverting this data set is shown in Fig. 5(b). It can be seen that patches separated by just $1 \mathrm{~km}$ are resolved in Fig. 5(b). This is consistent with the theoretical resolution predicted by use of expression (20).

\section{Conclusions and Refinements}

The methods outlined and the results obtained from the numerical simulations support the feasibility of a Doppler-only approach to radar mapping of the poles of the Moon or a planet. The simulation results indicate that, for an orbital altitude of $150 \mathrm{~km}$ and a receiver temperature of $1000 \mathrm{~K}$, a radar power of 10 $\mathrm{W}$ gives a signal-to-noise ratio sufficient for the spatial reflectivity distribution to be recovered.

In addition to its use as a demonstrator of feasibility, the simulation and inversion codes could be used during the mission-design stage to determine the effects of varying mission parameters (e.g., orbital altitude, transmitter power, receiver noise) on the quality of the images that are obtained. A number of refinements could be investigated.

\section{A. Sphericity}

It has been assumed that the polar region of the target body is a plane surface. Introducing the effects of sphericity would have two consequences. First, the Doppler strips would no longer be hyperbolas. With the same reasoning that was used to convert from the straight-line case to the hyperbola case, this effect could easily be accounted for in the inversion algorithm. Second, the geometrical factors would have to be modified. Sphericity, however, would introduce only circularly symmetric factors that could be divided out of the final image.

\section{B. Other Inversion Algorithms}

The Nievergelt convolution kernel is by no means the only function that can be used when the Radon inversion formula is approximated. Experimentation with other regularizing families of functions may yield improved results. There are also other inversion algorithms besides the convolution method. Algebraic reconstruction techniques ${ }^{11}$ could incorporate arbitrary Doppler strips and a nonsymmetric weighting function in the primary inversion algorithm. The quality of output and the computational efficiency of all projection inversion methods should be investigated with respect to this application.

\section{Multibeam Inversion}

Data obtained when the beam is centered at a point before the pole could be combined with data obtained from a point after the pole to produce a data set with an effective weighting function that consists of two individual weighting functions juxtaposed along the spacecraft track. This highly noncircular weighting function could then be corrected for use with the $\mathrm{Ku}$ nyansky algorithm. This method could extend the area mapped to several beam diameters away from the pole, although a degradation of resolution should be expected as distance from the pole increases. Accurately combining the data taken at different points along the track may also be problematic.

\section{References}

1. N. J. S. Stacy, "High resolution synthetic aperture radar observations of the Moon," Ph.D dissertation (Cornell University, Ithaca, N.Y., 1993).

2. J. K. Harmon, and M. A. Slade, "Radar mapping of Mercury: full-disk images and polar anomalies," Science 258, 640-643 (1992).

3. R. F. Jurgens, M. A. Slade, and R. S. Saunders, "Evidence for highly reflecting materials on the surface and subsurface of Venus," Science 240, 1021-1023 (1988).

4. D. O. Muhleman, B. J. Butler, A. W. Grossman, and M. A. Slade, "Radar images of Mars," Science 253, 1508-1513 (1991). 
5. R. Gens, and J. L. Vangenderen, "SAR interferometry-issues, techniques, applications,” Int. J. Remote Sensing 17, 18031835 (1996).

6. W. T. K. Johnson, "Magellan imaging radar mission to Venus," Proc. IEEE 79, 777-790 (1991).

7. J. H. Thomson and J. E. B. Ponsonby, "Two-dimensional aperture synthesis in lunar radar astronomy," Proc. R. Soc. London Ser. A 303, 477-491 (1968).

8. J. H. Taylor, "Two-dimensional brightness distributions of radio sources from Lunar occultation observations," Astrophysics 150, 421-426 (1967).

9. D. O. Muhleman, "Radar scattering from Venus and the Moon,” Astron. J. 69, 34-41 (1964).

10. T. S. Durrani and D. Bisset, "The Radon transform and its properties," Geophysics 49, 1180-1187 (1984).

11. G. T. Herman, Image Reconstruction from Projections (Academic, New York, 1980).

12. Y. Nievergelt, "Elementary inversion of Radon's transform," SIAM Rev. 28, 79-84 (1986).

13. J. Radon, "Uber die Bestimmung von Funktionen durch ihre Integrralwerte langs gewisser Mannigfaltigkeiten," Ber. Verhb. Saechs. Akad. Wiss. Leipzig Math. Phys. Kl. 69, 262277 (1917).

14. L. A. Kunyansky, "Generalized and attenuated Radon transforms: restorative approach to the numerical inversion," Inverse Probl. 8, 809-819 (1992).

15. S. J. Ostro, "Planetary radar astronomy," Rev. Mod. Phys. 65, 1235-1279 (1993). 